\title{
Factors associated with ventilator-associated events: an international multicenter prospective cohort study
}

\author{
Jordi Rello ${ }^{1,2,3}$ - Sergio Ramírez-Estrada ${ }^{4,5}$ - Anabel Romero ${ }^{1,2} \cdot$ Kostoula Arvaniti $^{3,6}$ - Despoina Koulenti ${ }^{3,7,8,9}$. \\ Saad Nseir ${ }^{10}$ • Nefise Oztoprak ${ }^{11}$ • Lila Bouadma ${ }^{12}$ • Loreto Vidaur ${ }^{13}$ • Leonel Lagunes ${ }^{3,14}$ - Yolanda Peña-López ${ }^{2,15}$. \\ for the EUVAE Study Group
}

Received: 27 March 2019 / Accepted: 17 May 2019 / Published online: 24 June 2019

(C) Springer-Verlag GmbH Germany, part of Springer Nature 2019

\begin{abstract}
A secondary analysis of a prospective multicenter cohort was performed in six intensive care units (ICU) in four European countries (France, Greece, Spain and Turkey). The main objective was to identify factors associated with ventilator-associated events (VAEs) in adults who underwent mechanical ventilation $(M V) \geq 48 \mathrm{~h}$. Secondary objectives were to identify: variables influencing VAE in the subpopulation with endotracheal intubation and in those subjects who were ventilated $>7$ days. Subjects who had undergone $M V \geq 48 \mathrm{~h}$ were included. In subjects with multiple episodes of MV, only the first one was eligible. The adult definitions for VAEs were adjusted to the 2015 update of the CDC's 2013 National Healthcare Safety Network Association. Factors associated with VAE were estimated through multivariate Cox proportional hazards analysis. Among 163 adults (42 tracheostomies), 76 VAEs (34.9 VAEs/1,000 ventilator-days) were documented: 9 were Ventilator-Associated Conditions (VAC) and 67 Infection-related Ventilator-Associated Complications (IVAC)-plus (9 only IVAC and 58 Possible Ventilator-Associated Pneumonia). VAEs developed after a median of 6 days (interquartile range: 4-9). VAEs were independently associated with longacting sedative/analgesic drugs (Hazard Ratio [HR]: 4.30), selective digestive decontamination (SDD) (HR: 0.38), and surgical/ trauma admission (HR: 2.30). Among 116 subjects with endotracheal tube, SDD (HR: 0.21 ) and surgical/trauma admission (HR: 3.11) remained associated with VAE. Among 102 subjects ventilated $>7$ days, only long-acting sedative/analgesic agents (HR: 8.69) remained independently associated with VAE. In summary, SDD implementation and long-acting analgesic/sedative agents restriction prescription may prevent early and late VAEs, respectively. Bundles developed to prevent VAEs should include these two interventions.
\end{abstract}

Keywords Ventilator-associated pneumonia - Selective digestive decontamination - Midazolam · Prevention bundles · Mechanical ventilation $\cdot$ Safety

\section{Introduction}

The implementation of ventilator bundles should be based on variables that influence the risk of ventilator-associated events (VAEs) [1]. Few studies have defined the risk factors for VAEs

Electronic supplementary material The online version of this article (https://doi.org/10.1007/s10096-019-03596-x) contains supplementary material, which is available to authorized users.

Anabel Romero

arm.anabel@gmail.com

Extended author information available on the last page of the article or how best to prevent these events. As healthcare systems differ according to geographical region, the composition of the bundles proposed is not the same in the USA, a country where private practice predominates, and in the European Union, with its national health care systems [1-3]. Certain practices such as selective digestive decontamination (SDD) [4] also present regional variations. Thus, potential new ventilator bundles should be based on evidence derived from analyses of associations between VAEs and clinical practice. The variability in clinical practice provides an opportunity to identify potential areas that might benefit from intervention.

Recently, two prospective studies with a similar design [5, 6] carried out by our group have described VAEs in adults and children undergoing mechanical ventilation (MV). Subjects recruited at six different sites from Europe were studied in 
order to identify variables associated with a first VAE. Our hypothesis was that many variables would be suitable for intervention. Our main objective was to identify factors associated with VAEs in patients who underwent MV $\geq 48 \mathrm{~h}$. Secondary objectives were to identify variables influencing VAE in the subpopulation with endotracheal intubation, and in patients who underwent MV during more than 7 days.

\section{Methods}

\section{Study design and inclusion criteria}

The study was a secondary analysis of a prospective, international, multicenter study [6]. Thirty consecutive subjects who had undergone MV for $48 \mathrm{~h}$ or more were eligible. Sites reporting less than two VAE episodes or less than two controls were excluded (Figure 1 of the electronic supplementary material). Subjects with ICU-acquired respiratory viral infections were also excluded. Daily follow-up was implemented for 30 days. Duration of MV was considered until extubation or ICU death. In patients with more than one episode of MV, only the first one was considered. Data were collected through a collaborative web database (http://compartint.net/euvae/). An external quality assessment was performed for severityof-illness score in a 5\% sample of variables, which reported an agreement above $83 \%$.

\section{Definitions}

An episode of ventilation was defined by the number of consecutive days during which the patient was ventilated. A period of at least one calendar day off the ventilator, followed by re-initiation of ventilation, defined a new episode of ventilation. The adult definitions for ventilator-associated events were adjusted to the 2015 update [7] (Fig. 1) of the CDC's 2013 National Healthcare Safety Network [8]. Delirium definition was reported elsewhere [9].

\section{Study variables}

The following patients' characteristics were considered: gender, age, presence of comorbidities, body mass index, and severity-of-illness index. High severity was defined as an APACHE II score above 20. Medical, surgical, and trauma patients were included.

Reasons for intubation included the following: respiratory failure, surgery, cardiogenic shock, altered level of consciousness, and sepsis/septic shock. Intubation could be performed in the ICU or elsewhere (operation room, pre-hospital, emergency department or hospital ward). Other variables recorded were implementation of SDD, chlorhexidine rinse $(0.12 \%$, $0.2 \%$ or $2 \%$ concentrations) for oral care, spontaneous breath trials, pharmacological paralysis, early mobility, tracheostomy, vasopressors, red blood cell units transfused, delirium, type of sedation (continuous or intermittent), drugs for sedation (midazolam, propofol), as well as drugs for analgesia (fentanyl, morphine, remifentanyl). Analgesic and sedative drugs were classified as follows: long-acting drugs (midazolam, fentanyl, morphine) and short-acting drugs (propofol, remifentanyl).

The following patients' outcomes were considered: length of stay (ICU and hospital) and ICU mortality.

\section{Statistical analysis}

A descriptive analysis was performed for patient characteristics, reporting percentages and medians, with its interquartile ranges (IQR) for quantitative variables. Two measures of VAE incidence were computed: (1) number of VAEs divided by the total number of ventilator-days and (2) number of VAEs divided by the total number of episodes of MV. To compare proportions, we used the Pearson's $X^{2}$ test, whereas to compare medians of quantitative variables (length of stay, ICU and hospital) we used the Mann-Whitney $U$ test.

Association between factors and VAEs was estimated using Cox proportional hazards regression models. Hazard ratios (HR) and 95\% confidence intervals (CI) were computed. Three multivariate models were constructed, according to the characteristics of patients included: (1) overall population, (2) patients with endotracheal tube, and (3) patients who underwent MV during more than 7 days. A fourth multivariate model was constructed to assess the association between factors and IVAC-plus. Patients were censored on extubation or death. We considered time-dependent variables for daily sedative and analgesic drugs, spontaneous breath trials and pharmacological paralysis. All multivariate analyses were adjusted by center. Variables were considered for the multivariate model if they reached a $p$ value of 0.10 in the univariate Cox proportional hazards regression models. Statistical significance was considered if the $p$ value was $<0.05$. All statistical analyses were performed using the SPSS Statistics version 25.0 (IBM).

\section{Results}

The study population included 163 patients undergoing MV (2,178 ventilator-days) for at least $48 \mathrm{~h}$ at 6 intensive care units (ICU) in 4 European countries (France, Greece, Spain and Turkey). Forty-two (25.8\%) underwent a tracheostomy. Males $(n=103,63.2 \%)$ were predominant. Median age was 60 years (IQR: 48-72) and median APACHE II Score was 21 (IQR: 14-26). Patients' characteristics are summarized in Table 1. Acute respiratory failure was the cause of intubation in $79(49.1 \%)$ patients. The presence of two or more 


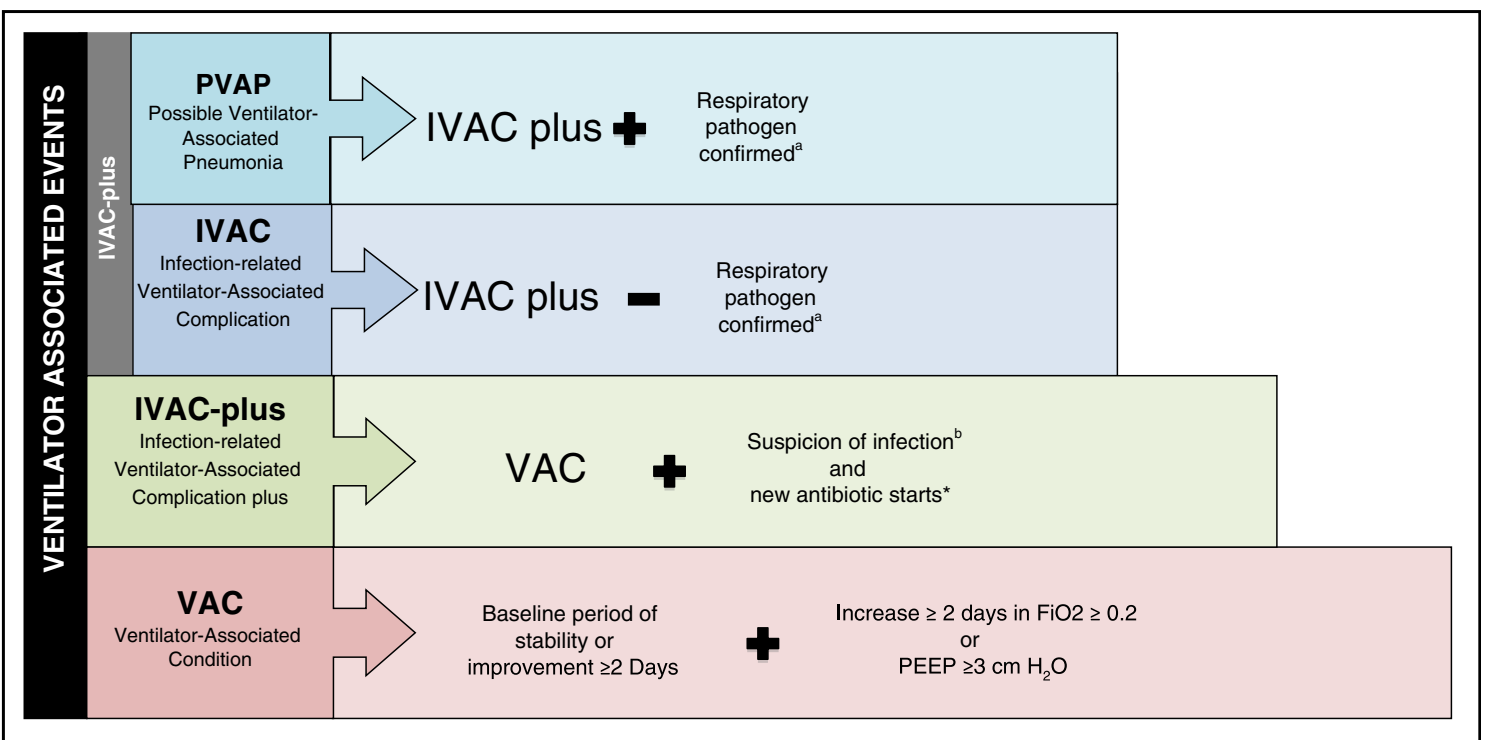

Fig. 1 Ventilator-associated events definitions. *The new antimicrobial agent must be started and sustained for at least 4 calendar days. ${ }^{a}$ Respiratory pathogen confirmation: one of the following: (1) positive culture of respiratory samples meeting quantitative or semi-quantitative thresholds or (2) insufficient growth of a pathogenic microorganism in respiratory samples plus purulent sputum $(>25$ neutrophils and $\leq 10$ squamous epithelial cells per low power field) or (3) organisms identified from pleural fluid specimen, positive tests for Legionellas species, or positive lung histopathology. ${ }^{\mathrm{b}}$ Suspicion of infection: fever $\left(\geq 38^{\circ} \mathrm{C}\right)$ or hypothermia $\left(\leq 36{ }^{\circ} \mathrm{C}\right)$ or leukocytosis $(\geq 12,000$ cells $/ \mathrm{mL})$ or leukopenia $(\leq 4,000$ cells $/ \mathrm{mL})$ comorbidities was identified in 51 patients $(32.7 \%)$. Of 76 VAEs (34.9 per 1,000 ventilator-days, 46.6 per 100 episodes), 9 were Ventilator-Associated Conditions (VAC) and 67 Infection-related Ventilator-Associated Complications (IVAC)-plus (9 only IVAC and 58 Possible VentilatorAssociated Pneumonia). Median day of VAE onset was day 6 (IQR: 4-9), with $51(67.1 \%)$ presenting within the first 7 days of MV. IVAC-plus (the sum of IVAC and PVAP) represented 44 out of 52 VAEs (84.6\%) within the first 7 days of ventilation compared with 23 out of 24 VAEs (95.8\%) 7 days or more after MV $(p=0.258)$. Fifty of 163 patients $(30.7 \%)$ died. VAEs were significantly more common among patients who died $(58.0 \%$ vs $41.6 \%, p=0.05)$. Most ICU deaths occurred early during hospitalization (median 6 days, IQR: 5-9), $36(72.0 \%)$ of them during the first 15 days. In survivors, median length of stay was significantly higher, both for ICU (29 vs $13 p<0.05$ ) and for hospital (48 vs $23 p<0.05$ ) in patients developing VAEs.

Factors associated with VAE, adjusted by center, are reported in Table 2. In the overall population, a VAE was more likely to occur in trauma or surgical patients (HR: 2.30) than in medical patients. Long-acting drugs prescription (HR: 4.30) was identified as a risk factor for VAE, whereas the use of SDD was identified as a protective factor for VAE (HR: $0.38)(p<0.05)$. In subjects with endotracheal tube, surgical or trauma admission was also identified as a risk factor for developing a VAE (HR: 3.11); again, SDD was identified as a protective factor (HR: 0.21$)(p<0.05)$. In patients under MV during more than 7 days, the prescription of long-acting drugs was identified as a risk factor for developing a VAE (HR: 8.69) $(p<0.05)$. Factors associated with IVAC-plus are reported in Table 3 of the electronic supplementary material section. SDD was identified as a protective factor for developing an IVAC-plus (HR: 0.31) and long-acting drugs prescription was identified as a risk factor for IVAC-plus (HR: 3.83$)(p<0.05)$. Distribution of VAE incidences, time to VAE, duration of MV, SDD use, type of patient, and the use of long-acting drugs, according to each participant ICU, is detailed in Table 4 of the electronic supplementary material.

\section{Discussion}

This study of a multicenter cohort of mechanically ventilated subjects is the first to assess variables potentially associated with VAEs. VAEs were common, being PVAP six times more frequent than IVAC alone, which emphasizes the relevance of efforts to prevent respiratory infections. Interestingly, both SDD implementation and long-acting sedative/analgesic agents use, variables that are amenable to intervention, significantly influenced VAEs, and their influence remained significant when subjects with tracheostomy were excluded. Lastly, our study confirms the association of VAEs with worse outcomes.

Our findings indicate that compliance with SDD can influence the risk of developing VAEs. The high proportion of IVAC-plus in our cohort may explain why SDD implementation independently reduced VAE rates in subjects submitted to 
Table 1 Patients' characteristics

\begin{tabular}{|c|c|c|c|}
\hline Characteristic & $\begin{array}{l}\text { Total } \\
N(\%) \\
163(100.0)\end{array}$ & $\begin{array}{l}\text { VAE } \\
N(\%) \\
76(46.6)\end{array}$ & $\begin{array}{l}\text { No VAE } \\
N(\%) \\
87(53.4)\end{array}$ \\
\hline \multicolumn{4}{|l|}{$\operatorname{Sex} N=163$} \\
\hline Female & $60(36.8)$ & $23(38.3)$ & $37(61.7)$ \\
\hline Male & $103(63.2)$ & $53(51.5)$ & $50(48.5)$ \\
\hline Age $^{*} N=163$ & $60(48-72)$ & $62(49-73)$ & $59(46-68)$ \\
\hline$\leq 40$ & $32(19.6)$ & $12(37.5)$ & $20(62.5)$ \\
\hline $41-60$ & $50(30.7)$ & $23(46.0)$ & $27(54)$ \\
\hline$>60$ & $81(49.7)$ & $41(50.6)$ & $40(49.4)$ \\
\hline APACHE score* $N=160$ & $21(14-26)$ & $20(12-24)$ & $23(15-29)$ \\
\hline High severity (>20) & $83(51.9)$ & $34(41)$ & $49(59)$ \\
\hline Low severity $(\leq 20)$ & $77(48.1)$ & $40(51.9)$ & $37(48.1)$ \\
\hline \multicolumn{4}{|l|}{ Comorbidities $N=156$} \\
\hline$\leq 1$ & $105(67.3)$ & $54(51.4)$ & $51(48.6)$ \\
\hline$\geq 2$ & $51(32.7)$ & $22(43.1)$ & $29(56.9)$ \\
\hline \multicolumn{4}{|l|}{ Reason of intubation $N=161$} \\
\hline Respiratory failure & $79(49.1)$ & $35(44.3)$ & $44(55.7)$ \\
\hline Other & $82(50.9)$ & $39(47.6)$ & $43(52.4)$ \\
\hline Surgery & $25(15.5)$ & $15(60.0)$ & $10(40)$ \\
\hline Cardiogenic shock & $2(1.2)$ & $1(50.0)$ & $1(50)$ \\
\hline Altered level of consciousness & $41(25.5)$ & $20(48.8)$ & $21(51.2)$ \\
\hline Sepsis. Septic shock & $14(8.7)$ & $3(21.4)$ & $11(78.6)$ \\
\hline \multicolumn{4}{|l|}{ Place of intubation $N=163$} \\
\hline Intensive care unit & $76(46.6)$ & $35(46.1)$ & $41(53.9)$ \\
\hline Other & $87(53.4)$ & $41(47.1)$ & $46(52.9)$ \\
\hline Anesthesia/operating room & $30(18.4)$ & $19(63.3)$ & $11(36.7)$ \\
\hline Pre-hospital & $22(13.5)$ & $5(22.7)$ & $17(77.3)$ \\
\hline Emergency department & $17(10.4)$ & $11(64.7)$ & $6(35.3)$ \\
\hline Hospital ward & $18(11.0)$ & $6(33.3)$ & $12(66.7)$ \\
\hline \multicolumn{4}{|l|}{ Type of patient $N=163$} \\
\hline Medical & $101(62.0)$ & 37 (36.6) & $64(63.4)$ \\
\hline Other & $62(38.0)$ & $39(62.9)$ & $23(37.1)$ \\
\hline Surgical & $47(28.8)$ & $28(59.6)$ & $19(40.4)$ \\
\hline Trauma & $15(9.2)$ & $11(73.3)$ & $4(26.7)$ \\
\hline
\end{tabular}

$V A E$ ventilator-associated event, $A P A C H E$ acute physiology and chronic health disease classification system *median and interquartile range

Table 2 Factors associated with VAE: multivariate cox proportional hazards models

\begin{tabular}{|c|c|c|c|}
\hline \multirow[t]{2}{*}{ Factor } & \multicolumn{3}{|l|}{$\mathrm{HR}(95 \% \mathrm{CI})$} \\
\hline & Overall $(N=163)$ & Endotracheal tube $(N=116)$ & MV $>7$ days $(N=102)$ \\
\hline Type of patient, surgical, or trauma & $2.30(1.04-5.1)$ & $3.11(1.04-9.32)$ & $1.68(0.69-4.1)$ \\
\hline SDD & $0.38(0.15-0.92)$ & $0.21(0.06-0.72)$ & $0.43(0.14-1.26)$ \\
\hline Tracheostomy & $0.88(0.39-2.01)$ & - & $0.75(0.3-1.89)$ \\
\hline Long-acting drugs ${ }^{*}$ & $4.30(1.62-11.42)$ & $2.56(0.77-8.54)$ & $8.69(2.53-29.86)$ \\
\hline
\end{tabular}

$V A E$ ventilator-associated event, $C I$ confidence interval, $H R$ hazard ratio, $M V$ mechanical ventilation, $S D D$ selective digestive decontamination

* Long-acting drugs: midazolam, fentanyl, and morphine. Results in bold are statistically * significant, $\mathrm{p}<0.05$ 
long periods of MV. Another explanation for the effect of SDD on VAE is that much of VAE consists of tracheal colonization combined with a non-infectious pulmonary pathology such as pleural effusion, atelectasis, or pulmonary edema. Preventing tracheal colonization is the mechanism by which many VAP-prevention strategies such as subglottic suction, silver impregnated tubes, and oral decontamination work.

Moreover, our results also identify long-acting sedatives/ analgesic agents as an independent risk factor for VAEs, rather than delirium, suggesting that intervention in sedative/ analgesic prescription might be a possible strategy of prevention. This may be related with a higher probability of impregnation when prescribing drugs prone to being accumulated, and also with an underestimation of delirium if a systematic delirium-screening instrument is not used for its diagnosis in the ICU. If only clinical criteria are applied, it is the hyperactive subtype that will usually be detected [10]. However, recent data show that most delirious ICU patients have hypoactive delirium, which is more frequent in patients with more severe illness and undergoing MV [11]. Thus, the spontaneous breathing trial was not found to protect against VAE in our cohort. These findings are in line with the association between benzodiazepines and increased MV duration and ICU stay compared with other sedatives, as described in two recent meta-analyses [12, 13] and a cohort study [14]. Although midazolam may be considered as a short-acting benzodiazepine, its pharmacokinetic profile, with phase 1 and phase 2 metabolism and an end-active metabolite with renal excretion, frequently results in accumulation, over-sedation, and delayed wakening in the ICU, particularly in older and sicker patients, as it has been pointed out by Wyncoll et al. [15]. When analyzed midazolam in conjunction with morphine and fentanyl, those continuous around-the-clock drugs resulted the most important factor for developing VAE.

In the coming years, it would be interesting to analyze the role of alpha-2 agonists, which were hardly used in Europe during our study recruitment period [16], and the impact of new non-benzodiazepine sedation approach and ICU delirium guidelines on VAE [17-19]. In recent years, there has been an increasing concern with delirium in the ICU and its impact on patient safety and outcomes, and indeed delirium has been included in some evidence-based interventions designed to reduce adverse events in hospitals [20]. On the other hand, the difficulty of ventilator-associated pneumonia (VAP) diagnosis and the discrepancies in antibiotic consumption despite reporting/achieving low VAP rates [21] have shifted the focus of quality-improvement on patient outcomes beyond the mere recording of VAP rates in ventilated patients, and the concept of VAE has emerged.

Conservative rather than liberal fluid resuscitation will increase the number of ventilator-free days and several studies $[14,22]$ have reported a relation between VAEs and excess fluids. However, our data did not find this association, perhaps due to differences in case mix, comorbidities (cardiomyopathy), VAC rates or measurement of fluid overload, or because the other studies limited their assessment to the first 4 days of ICU stay [14]. Other studies of potentially modifiable VAE risk factors [14, 22-26] have identified blood transfusions and mandatory modes of MV with high inspiratory pressure.

This study has several limitations. First, in spite of the multicenter design, the fact that only a few events per site were analyzed limits its power to identify potential risk factors. Second, dexmedetomidine was not properly evaluated due to its low use in the ICUs during the period of study, and further studies are needed to identify its potential effect. Third, this study was conducted in Europe and the interpretations may not be applicable to other settings, due to the large variation in therapeutic strategies, and duration of MV. Fourth, some potentially relevant variables such as average gastric retention were not evaluated. Five, the number of trauma patients is small and variables can be different in other case-mixes. Lastly, we considered the entire period of MV: other variables may be relevant if the time span is limited, for instance to the first 4 days of ventilation.

\section{Conclusions}

Future bundles should include SDD implementation and longacting analgesic/sedative agent restriction as potential strategies to prevent VAEs.

Acknowledgments We thank Michael Maudsley for providing assistance with English language corrections to this manuscript. We also thank Santiago Pérez-Hoyos from the Statistics and Bio informatics Unit (UEB) at Vall d'Hebron Research Institute (VHIR) for giving statistical advice. This research was carried out as part of a $\mathrm{PhD}$ program in Health Science at the Universitat Autónoma de Barcelona, Spain, and was supported by the European Society of Clinical Microbiology and Infectious Diseases (ESCMID) Study Group for Infections in Critically Ill Patients (ESGCIP), Basel, Switzerland, and by Centro de Investigación Biomédica en Red - CIBERES CB06/06/036, Madrid, Spain.

We thank all EUVAE collaborators for collecting the data or scientific advice: Benito Almirante (Hospital Universitari Vall d'Hebron, Barcelona, Spain), Aliye Bastug (Ankara Numune Training and Research Hospital, Turkey), Mateo Bassetti (Santa Maria Misericordia Hospital, University of Udine, Udine, Italy), Ilkay Bozkurt (Ondokuz Mayis University, Samsun, Turkey), George Dimopoulos (Attikon University Hospital, Athens, Greece), Asuman Inan (Haydarpasa Numune Hospital, Istanbul, Turkey), Marina Oikonomou (Papageorgiou General Hospital, Thessaloniki, Greece), Garyphallia Poulakou (Attikon University Hospital, Athens, Greece), David Thompson (RBWH), Estefania Torrivilla (RBWH), Izarne Totorika (Hospital Universitario de Donostia, Donostia, Spain), and Vipin Varghese (RBWH).

Funding Supported in part by grants from the European Society of Clinical Microbiology and Infectious Diseases-ESCMID, Study Group for Infections in Critically Ill Patients-ESGCIP, Basel, Switzerland, and by Centro de Investigación Biomédica en RedCIBERES 06/06/036, Instituto Salud Carlos III, Madrid, Spain, and Fundació Catalana de Pneumologia—FUCAP, Barcelona, Spain. 


\section{Compliance with ethical standards}

Conflict of interest Dr. Rello has served on the speakers' bureau and as a consultant for Cubist, Bayer, ROCHE, Medimmune, Pfizer, Anchoagen, and Aridis. The other authors report no conflicts of interest relevant to this article.

Ethical approval/informed consent The study protocol was approved by the institutional review board on human research at each participating center and at Vall d'Hebron Barcelona Hospital Campus, the coordinating center [PR(AG)25/2014]. Patients (or the relatives of unconscious patients) were asked to provide written consent prior to participation in the study.

\section{References}

1. Klompas M, Li L, Kleinman K, Szumita PM, Massaro AF (2016) Associations between ventilator bundle components and outcomes. JAMA Intern Med 176(9):1277-1283

2. Rello J, Afonso E, Lisboa T, Ricart M, Balsera B, Rovira A et al (2013) A care bundle approach for prevention of ventilatorassociated pneumonia. Clin Microbiol Infect 19(4):363-369

3. [No Authors listed] (2005) IHI proposes six patient safety goals to prevent 100,000 annual death. Qual Lett Healthc Lead 17(1):11-2, 1

4. Wittekamp BH, Plantinga NL, Cooper BS, Lopez-Contreras J, Coll P, Mancebo J et al (2018) Decontamination strategies and bloodstream infections with antibiotic-resistant microorganisms in ventilated patients: a randomized clinical trial. JAMA 320(20):20872098

5. Peña-López Y, Pujol M, Campins M, Lagunes L, Balcells J, Rello J (2018) Assessing prediction accuracy for outcomes of ventilatorassociated events and infections in critically ill children: a prospective cohort study. Clin Microbiol Infect 24(7):732-737

6. Ramírez-Estrada S, Lagunes L, Peña-López Y, Vahedian-Azimi A, Nseir S, Arvaniti K et al (2018) Assessing predictive accuracy for outcomes of ventilator-associated events in an international cohort: the EUVAE study. Intensive Care Med 44(8):1212-1220

7. CDC's National Healthcare Safety Network (2015) Ventilatorassociated event (VAE). Available at: https://www.cdc.gov/nhsn/ pdfs/pscmanual/10-vae final.pdf. Accessed 14 January 2019

8. Magill S, Rhodes B, Klompas M (2014) Improving ventilatorassociated event surveillance in the national healthcare safety network and addressing knowledge gaps: update and review. Curr Opin Infect Dis 27(4):394-400

9. Ramsay MA, Savege TM, Simpson BR, Goodwin R (1974) Controlled sedation with alphaxalone-alphadolone. Br Med J 2(5920):656-659

10. Spronk PE, Riekerk B, Hofhuis J, Rommes JH (2009) Occurrence of delirium is severely underestimated in the ICU during daily care. Intensive Care Med 35(7):1276-1280

11. Krewulak KD, Stelfox HT, Leigh JP, Ely EW, Fiest KM (2018) Incidence and prevalence of delirium subtypes in an adult ICU: a systematic review and meta-analysis. Crit Care Med 46(12):20292035

12. Zhang Z, Chen K, Ni H, Zhang X, Fan H (2017) Sedation of mechanically ventilated adults in intensive care unit: a network meta-analysis. Sci Rep 7:44979
13. Wang H, Wang C, Wang Y, Tong H, Feng Y, Li M et al (2018) Sedative drugs used for mechanically ventilated patients in intensive care units: a systematic review and network meta-analysis. Curr Med Res Opin 35(3):435-446 1-12

14. Liu J, Zhang S, Chen J, Mao Y, Shao X, Li Y, et al. (2018) Risk factors for ventilator-associated events: a prospective cohort study. Am J Infect Control

15. Wyncoll D, McKenzie C (2010) Sedation versus no sedation in the intensive-care unit. Lancet 375(9721):1159 author reply 1160

16. Weatherall M, Aantaa R, Conti G, Garratt C, Pohjanjousi P, Lewis MA et al (2017) A multinational, drug utilization study to investigate the use of dexmedetomidine (Dexdor $\left.{ }^{\circledR}\right)$ in clinical practice in the EU. Br J Clin Pharmacol 83(9):2066-2076

17. Barr J, Fraser GL, Puntillo K, Ely EW, Gélinas C, Dasta JF et al (2013) Clinical practice guidelines for the management of pain, agitation, and delirium in adult patients in the intensive care unit. Crit Care Med 41(1):263-306

18. Barr J, Kishman CP, Jaeschke R (2013) The methodological approach used to develop the 2013 Pain, Agitation, and Delirium Clinical Practice Guidelines for adult ICU patients. Crit Care Med 41(9 Suppl 1):S1-S15

19. Trogrlić Z, van der Jagt M, Lingsma H, Gommers D, Ponssen HH, Schoonderbeek JFJ et al (2019) Improved guideline adherence and reduced brain dysfunction after a multicenter multifaceted implementation of ICU delirium guidelines in 3,930 patients. Crit Care Med 47(3):419-427

20. Zegers M, Hesselink G, Geense W, Vincent C, Wollersheim H (2016) Evidence-based interventions to reduce adverse events in hospitals: a systematic review of systematic reviews. BMJ Open 6(9): 012555

21. Nora D, Póvoa P (2017) Antibiotic consumption and ventilatorassociated pneumonia rates, some parallelism but some discrepancies. Ann Transl Med 5(22):450

22. Lewis SC, Li L, Murphy MV, Klompas M, Prevention Epicenters CDC (2014) Risk factors for ventilator-associated events: a casecontrol multivariable analysis. Crit Care Med 42(8):1839-1848

23. Mekontso Dessap A, Katsahian S, Roche-Campo F, Varet H, Kouatchet A, Tomicic V et al (2014) Ventilator-associated pneumonia during weaning from mechanical ventilation: role of fluid management. Chest 146(1):58-65

24. Sim JK, Oh JY, Min KH, Hur GY, Lee SH, Lee SY et al (2016) Clinical significance of ventilator-associated event. J Crit Care 35: 19-23

25. Harris BD, Thomas GA, Greene MH, Spires SS, Talbot TR (2018) Ventilator bundle compliance and risk of ventilator-associated events. Infect Control Hosp Epidemiol 39(6):637-643

26. Guess R, Vaewpanich J, Coss-Bu JA, Phongjitsiri S, Kennedy C, Starke J et al (2018) Risk factors for ventilator-associated events in a PICU. Pediatr Crit Care Med 19(1):e7-e13

Publisher's note Springer Nature remains neutral with regard to jurisdictional claims in published maps and institutional affiliations. 


\section{Affiliations}

Jordi Rello ${ }^{1,2,3}$. Sergio Ramírez-Estrada ${ }^{4,5}$ - Anabel Romero ${ }^{1,2}$ - Kostoula Arvaniti ${ }^{3,6}$. Despoina Koulenti ${ }^{3,7,8,9}$. Saad Nseir ${ }^{10} \cdot$ Nefise Oztoprak $^{11} \cdot$ Lila Bouadma $^{12} \cdot$ Loreto Vidaur $^{13} \cdot$ Leonel Lagunes $^{3,14} \cdot$ Yolanda Peña-López $^{2,15}$

1 Centro de Investigación Biomédica en Red-Enfermedades Respiratorias (CIBERES), Vall d'Hebron Research Institute, Ps Vall d'Hebron 119 — AMI 14th floor, 08035 Barcelona, Spain

2 Vall d'Hebron Research Institute (VHIR), Barcelona, Spain

3 European Society of Clinical Microbiology and Infectious Diseases - Study Group for Infections in Critically Ill Patients (ESGCIP-ESCMID), Basel, Switzerland

4 Intensive Care Department, Clinica Corachan, Barcelona, Spain

5 Medicine Department, Universitat Autònoma de Barcelona, Barcelona, Spain

6 Intensive Care Unit, Papageorgiou Hospital, Thessaloniki, Greece

7 Critical Care Department, Attikon University Hospital, Athens, Greece

8 UQ Centre for Clinical Research, Faculty of Medicine, The University of Queensland, Brisbane, Australia
9 Royal Brisbane Clinical Unit, Faculty of Medicine, The University of Queensland, Brisbane, Australia

10 Critical Care Center, Hospital Universitaire Lille, Lille, France

11 Department of Infectious Diseases and Clinical Microbiology Clinic, Antalya Education and Research Hospital, Antalya, Turkey

12 Medical and Infectious Diseases ICU, Bichat-Claude-Bernard Hospital, Assistance Publique-Hôpitaux de Paris, Paris, France

13 Intensive Care Department, Donostia Univertsitate Ospitalea/ CIBERES, Donostia, Spain

14 Intensive Care Department Hospital Especialidades Médicas, San Luís Potosí, Mexico

15 Paediatric Critical Care Department, Hospital Universitari Vall d'Hebron, Barcelona, Spain 